\title{
Rationale and design of a UK database for a rare cancer type: the GEM Registry for gastrointestinal stromal tumours
}

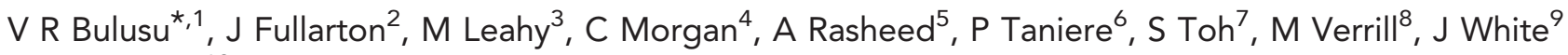 \\ and I Judson ${ }^{10}$ \\ ${ }^{1}$ Cambridge University Hospitals NHS Foundation Trust, Cambridge, UK; ${ }^{2}$ Strategen Ltd, Basingstoke, UK; ${ }^{3}$ Christie NHS \\ Foundation Trust, Manchester, UK; ${ }^{4}$ Velindre Cancer Centre, Cardiff, UK; ${ }^{5}$ Royal Gwent Hospital, Gwent Healthcare NHS Trust, \\ Newport, UK; ${ }^{6}$ Department of Cellular Pathology, University Hospitals Birmingham, Birmingham, UK; ${ }^{7}$ Queen Alexandra Hospital, \\ Portsmouth, UK; ${ }^{8}$ Freeman Hospital, Newcastle-upon-Tyne, UK; ${ }^{9}$ Beatson West of Scotland Cancer Centre, Glasgow, UK and \\ ${ }^{10}$ Royal Marsden NHS Foundation Trust, Surrey, UK
}

Background: Despite advances in the management of and changes in clinical practice, little is known about the epidemiology, patterns of care and outcomes of gastrointestinal stromal tumour (GIST) patients in the UK. Patient registries are receiving increasing attention as they can provide important information on clinical practice and patient outcomes. The rationale and study design of the GIST Epidemiology and Management (GEM) Registry, which forms part of the routine clinical practice for GISTs in several UK centres, are described.

Methods: The GEM Registry is a secure web-based registry system designed around a Microsoft Access core using SOL interface. Demographic, surgical, histopathological and clinical data will be captured including treatment outcomes and survival. The registry was piloted in six centres and following further fine tuning of the data sets, ethical committee submission and approval was completed.

Results: The GEM National Registry is the first of its kind to be implemented in rare cancers in UK. The registry is being rolled out initially in selected centres with the aim to expand to other centres. The first publication reporting analyses of the central data set is anticipated for the summer of 2013.

Conclusion: GEM Registry will enable us to obtain a clear picture of incidence/prevalence of GISTS in UK. Clinicians will be able to review the prognostic and predictive value of variables in a large prospective data set. The data can be used for planning the delivery and improving the quality of care. This information is likely to inform clinical practice and, in years to come, guide the development and implementation of clinical trials for novel tyrosine kinase inhibitors. The results will not only benefit the GIST community, but also serve as a basis for the study of other rare tumour types.

Gastrointestinal stromal tumour (GIST), the most common mesenchymal tumour of the gastrointestinal tract, accounts for $0.1-3 \%$ of all the gastrointestinal cancers (Miettinen and Lasota, 2001).They are clinically and biologically heterogeneous tumours and can occur throughout the gastrointestinal tract. The diagnosis of GIST is made on histological, immunohistochemical and molecular features. GISTs have a spindle cell/epithelioid morphology and express kit receptor in 95\% of cases, detected by CD 117 antibody. Discovered on GISTs-18 (DOG-1) is a specific and sensitive marker for GISTs. A typical GIST is CD 117 and DOG-1 
positive and desmin negative (Fletcher et al, 2002; West et al, 2004; Miettinen and Lasota, 2006a). Around $85 \%$ of GISTs have activating mutations in KIT or PDGFRA genes. The other $15 \%$, defined as 'wild type', may have alterations in BRAF, IGFR-1 and succinate dehydrogenase (Corless et al, 2011).

The median age of presentation of GISTs is in the seventh decade. GISTS are uncommon below the age of 40 years. Some studies have suggested a slight male predominance in adult sporadic GISTS, whereas paediatric and adolescent GISTS have a female preponderance (Kim et al, 2010; Call et al, 2012). The estimated annual incidence of GISTS is $11.5-15.0$ cases per million per year, but the true incidence may be higher (Kim et al, 2005; Nilsson et al, 2005; Tryggvason et al, 2005). GISTS most commonly arise from the stomach, followed by the small bowel, colon, rectum and oesophagus. Metastatic spread is often to the liver and peritoneum and less commonly to the bones and lungs (DeMatteo et al, 2000; Burkill et al, 2003).

The last two decades have witnessed a rapid progress in the understanding of the biology and treatment of GISTS. Constitutive activation of KIT has an important role in the growth and survival of GISTS (Hirota et al, 1998). This led to the rapid discovery of the tyrosine kinase inhibitor (TKI) STI 571 (imatinib) and the first patient was successfully treated in 2000 (Joensuu et al, 2001). Imatinib is now well established as first-line therapy for metastatic/ inoperable GISTS and the National Institute for Health and Clinical Excellence (NICE) approved its use for patients with KIT-positive unresectable/metastatic GISTS in 2004 (National Institute for Clinical Excellence, 2004; Heinrich, 2010). Second-line treatment with sunitinib in patients who are either intolerant to or progressed on imatinib has been shown to improve survival and was approved by NICE in 2009 (Demetri et al, 2006; National Institute for Health and Clinical Excellence, 2009). Resistance to TKIs is almost inevitable and there is desperate need for newer agents in patients with metastatic GISTS.

Despite the advances in the management of GISTs, little is known about the epidemiology, patterns of care and outcomes of GIST patients in the UK. There has been some progress in countries, for example in the USA, Poland, and Czech Republic, in gathering some tumour registry data (Brabec et al, 2009; Chacon et al, 2010; Pisters et al, 2011). NICE, as part of the technology assessment guidance issued in 2004 and 2009, has recommended that further data on the epidemiology, treatment and outcomes of GISTS should be captured in the UK (National Institute for Clinical Excellence, 2004; National Institute for Health and Clinical Excellence, 2009). Patient registries are receiving increasing attention as they can provide information on the referral pathways, clinical practice and treatment variations, and more importantly, on outcomes such as time to progression and overall survival (Gliklich and Dreyer, 2010).

The aim of this paper is to describe the rationale and study design of the GIST Epidemiology and Management (GEM) Registry, which forms part of the routine clinical practice for GISTs in several UK centres. The registry is designed to further characterise patients with GISTs and to provide comprehensive data to improve understanding of the incidence, treatment and outcomes of GISTs in the UK.

\section{MATERIALS AND METHODS}

Objectives. The primary objectives of the GEM Registry is to obtain robust estimates on the incidence, prevalence, recurrence and survival and mortality rates for GIST in the UK, and to record and analyse the progression of patients with advanced disease. The GEM Registry will also facilitate the optimisation of TKI therapy (e.g., relationship between mutation type and therapy), and elucidate longer-term benefits of TKI treatment (i.e., overall survival) in a UK population. The secondary objectives are to promote improvements in the treatment for GISTs by recording and analysing their clinical presentation; current treatment practices in the UK; whether the current prognostic criteria (Miettinen and Lasota, 2006b) are being used to determine risk stratification; and whether current treatment guidelines are being adhered to.

Overview of system. The GEM Registry is a web-based registry system that has the capacity to store a centre's data on patient characteristics, treatment and clinical outcomes on the local hospital server, or, alternatively, for centres treating fewer GIST patients, at the server maintained by the commercial host. Clinicians are able to access the system from anywhere by logging in via the hospital intranet. Periodically, at least monthly if new data are available, locally stored information is uploaded to the central UK GIST Registry (National Data set) held on the host server.

All centres that treat GIST patients will be invited to participate, and all patients, including paediatric patients with histologically confirmed or suspected GIST, are eligible for enrolment. Data from patients with suspected GIST will be captured, analysed and reported separately. The initial phase will be focusing on the centres represented by the authors with the aim to roll out to the other centres by 2014. Central pathology review will be undertaken as per standard UK GIST guidelines through the specialist multidisciplinary meetings at each centre. Six centres in the UK have been accredited to perform the mutational analysis. GISTs have a separate pathology SNOMED code that can be used to identify patients from smaller centres who may not be entered into the registry.

Database design. The GEM database has been designed around a Microsoft Access (MSACCESS) core using a SQL interface from specifically designed Active Server (asp) web pages (Figure 1). There are two main data input pages, for clinical (basic; Figure 2) and pathological (extended) data, together with facilities for reviewing historical records for each patient and generating realtime reports on the current database content. User access to the system is password protected and has multiple levels of privilege for data editing, record deletion, transmission to the central server, creation of new user accounts and so on. Wherever possible, the interface pages provide real-time assistance with data input, by providing reminders for mandatory fields, acceptable ranges for numeric fields, calendar support for dates and drop-down boxes for most text input.

Steering committee. The registry is regulated by the UK GEM Registry Steering Committee, comprised of recognised experts in GIST (please see authors). Addenbrooke's Hospital, Cambridge has been designated as the owner of the UK GEM Registry National Data set by consensus agreement of the Steering Committee. The Steering Committee reviews the requests for access to the registry. Each request is carefully reviewed on a case by case basis and

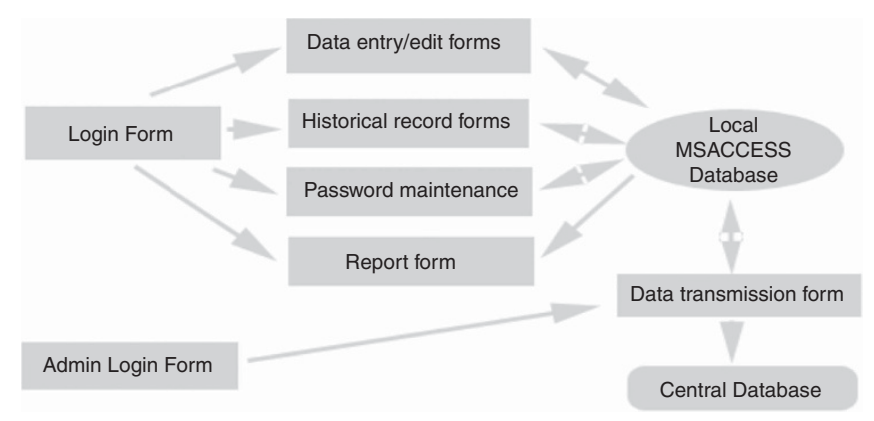

Figure 1. Schematic structure of the GEM Registry. 


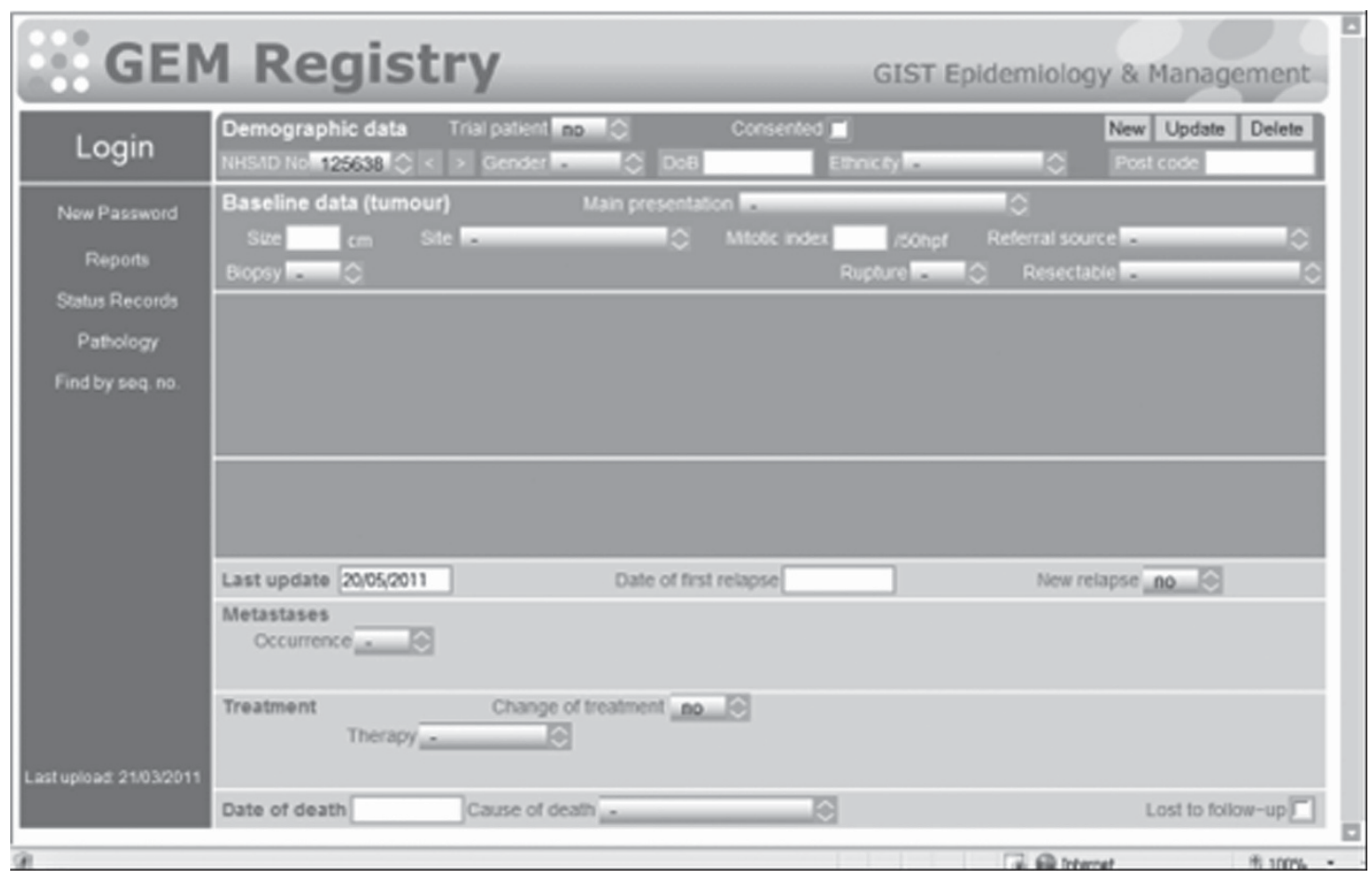

Figure 2. Main data input page of the GEM Registry.

\begin{tabular}{|l|}
\hline Table 1. Minimum data set \\
\hline Demographics \\
\hline Date of diagnosis \\
\hline Tumour characteristics \\
\hline Referral source \\
\hline Mode of presentation \\
\hline Biopsy details and date of procedure \\
\hline Rupture (yes/no) \\
\hline Risk assessment \\
\hline Tumour type \\
\hline Details of resection (if applicable) \\
\hline Adjuvant treatment \\
\hline Details of metastases (if applicable) \\
\hline Relapse date \\
\hline Participation in clinical trial (yes/no) \\
\hline Date and cause of death \\
\hline Consent received \\
\hline Loss to follow-up recorded \\
\hline
\end{tabular}

appropriate access granted for ethically approved research projects. The sponsor, Novartis Pharmaceuticals, has no automatic access to the registry or the biospecimens stored as part of the GIST biobank.

Data collection. All participating centres will capture a skeletal (minimum) data set for each patient (Table 1), which includes the demographics, tumour characteristics and date of entry into the registry. The data captured will be updated appropriately based on each patient's clinical management.

For those centres willing and able to, an extended data set is available to capture further pathological details (Table 2). All adverse events and any pregnancies occurring while on

\section{Table 2. Extended data set}

\begin{tabular}{|l|}
\hline Sample type (pre-treatment or post-treatment) \\
\hline Details of growth (endophytic or exophytic) \\
\hline Primary tumour with well-defined margin (yes/no) \\
\hline Tumour haemorrhage, necrosis and/or calcification \\
\hline Nodes affected (yes/no) \\
\hline Type of specimen used for diagnosis \\
\hline Histological features \\
\hline Mutation testing \\
\hline
\end{tabular}

treatment are to be captured and reported, as per standard clinical practice.

Ethical considerations. The UK GEM Registry will be implemented and reported in accordance with applicable local regulations and with the ethical principles laid down in the Declaration of Helsinki. Ethical approval has been granted centrally for the registry via the National Research Ethic Service, as there is intent to combine, collate, analyse and publish the data captured by the system. Eligible patients may only be included in the study after providing written, informed consent.

Piloting of the UK GEM Registry. The UK GEM Registry has been piloted on a secure, dedicated, development server, with clinicians treating GISTs from the major centres in the UK being provided a user name and password to access the system. Piloting has allowed GIST clinicians using the registry to test the software in situ and to test the various functions of the registry in order to identify any areas for improvement. Clinicians were instructed to refrain from using real patient data, to experiment with all fields and areas within the registry, to frequently revisit the web page to ensure updates and modifications were processed, and to then feedback any comments and/or modifications to the software. Any suggested modifications were agreed by the Steering Committee before implementation on the website. 
Data security and integrity. All data collection forms and data reports include the subject's local study identification number (NHS number or equivalent) and a unique UK GEM Registry sequence number. All data collected locally on individual patients will be transmitted centrally alongside this GEM Registry sequence number but without any other patient identifier, to ensure anonymity.

The GEM Registry is set up both locally and centrally with privilege levels appropriate to the status and seniority of the user. Access to the system is limited to individuals having access to the local intranet and governed by a personal user name and password. Reminders are automatically generated on a monthly basis for users to renew their password. An algorithm has been implemented to assess password strength in real time, and thereby assist the user to maximise security of the database. Only administrators with the highest level of privilege are permitted to add new user accounts and transmit data to the central server. Data transmission to and from the central server is predicated on the implementation of a password protected secure server protocol with data encryption.

Data clerks, nurses and clinicians at each participating centre have attended training sessions to ensure data accuracy. Every unit has had at least half a day's training on the use of the registry tool. In addition, training on various aspects of the registry has been supplemented by the provision of a user guide (Table 3 ). E-mail and telephone support has also been provided. There will be ongoing training and support for any newly recruited centres.

Drop-down boxes, calendars and numeric limits in the web-based software interface reduce the likelihood of human error. Periodic on-site quality assurance checks are maintained, together with continuous statistical comparisons of local data between centres to warrant data consistency. To ensure data are updated on a regular basis, user accounts identified as being inactive for prolonged periods will be issued automatic reminders to log into the system. The central system will continuously flag up multiple common fields between cases to eliminate the possibility of duplicated patient records.

Statistical analysis. Central analyses are carried out on a periodic basis, as new data are assembled. These analyses are designed to highlight the data shown in Table 4 in the aggregated and anonymised UK data set.

Current status. The UK GEM Registry has currently been implemented or is in the process of being implemented at 10 major GIST centres in the UK (including those represented by the authors). Following an interim review of the data at six months, the registry will be rolled out to the smaller centres. The first publication reporting analyses of the central data set is anticipated for the autumn 2013. A GIST biobank has been set up in 2012 as a separate project. Ethics approval has been granted. All registered patients will be invited to give informed consent for the tumour specimens to be stored. This has the full support of the patient community and is endorsed by GIST Support UK.

\section{DISCUSSION}

Although data on the epidemiology of GIST have advanced in recent years, comprehensive epidemiological data on GISTs in the UK are lacking. There is therefore a clear need-recognised by specialists throughout the UK-for a specific GIST registry. The information collected from the UK GEM Registry will provide important insights into the incidence, prevalence, recurrence, survival and mortality rates of GISTs, as well as treatment practices throughout the UK, thereby enabling therapeutic intervention to be evaluated and ultimately optimised. The registry will also enable clinicians to review the prognosis of different patient groups and
Table 3. Information in UK GEM Registry user guide

\section{Instructions or use}

Details of system functions

Definitions of all terms used in the registry system

How to report adverse events

How to transmit to and store data in the cental data set

Abbreviation: GEM = GIST Epidemiology and Management

\begin{tabular}{|l|}
\hline Table 4 . Data in the aggregated and anonymised UK data set \\
\hline Demographics \\
\hline Age \\
\hline Time from diagnosis to treatment \\
\hline Previous therapy and surgery \\
\hline Other prognostic factors \\
\hline Initial systemic therapy \\
\hline Drug/product \\
\hline Dose \\
\hline Dosage interval \\
\hline Impact on signs and symptoms \\
\hline Time to dose alteration \\
\hline Time to discontinuation \\
\hline Adverse events \\
\hline Disease progression \\
\hline Death \\
\hline Use of other resources/prognostic factors \\
\hline Global factors \\
\hline Incidence and prevelance \\
\hline Time to disease progression \\
\hline Time to death \\
\hline Trend in prognostic factors over time \\
\hline Subanalysis by tumour type (locally advanced, metastatic) \\
\hline
\end{tabular}

identify long-term therapeutic benefits of TKI treatment which have, to date, been difficult to estimate in the UK. In addition, the data can be used for planning and improving the quality of care delivery. This information may help to inform clinical practice and, in years to come, guide the development and implementation of clinical trials for novel TKIs. The insights gained from the web-based GEM Registry data will not only benefit individuals with GISTs, but also serve as a basis for the study of other rare tumour types.

\section{ACKNOWLEDGEMENTS}

Development of the UK GEM Registry and ongoing training was supported by an unrestricted educational grant from Novartis Pharmaceuticals UK limited. Strategen Limited developed the web pages and database for the GEM Registry and provided technical support to centres. RFM Limited provided hosting servers for the central data set. 


\section{CONFLICT OF INTEREST}

The authors declare no conflict of interest.

REFERENCES

Brabec P, Sufliarsky J, Linke Z, Plank L, Mrhalova M, Pavlik T, Klimes D, Gregor J (2009) A whole population study of gastrointestinal stromal tumours in the Czech Republic and Slovakia. Neoplasma 56(5): 459-464.

Burkill GJC, Badran M, Al-Muderis O, Thomas JM, Judson IR, Fisher C, Moskovic EC (2003) Malignant gastrointestinal stromal tumor: distribution, imaging features, and pattern of metastatic spread. Radiology 226(2): 527-532.

Call J, Walentas CD, Eickhoff JC, m Scherzer N (2012) Survival of gastrointestinal stromal tumor in the imatinib era: life raft group observational registry. BMC Cancer 12: 90.

Corless CL, Barnett CM, Heinrich MC (2011) Gastrointestinal stromal tumours: origin and molecular oncology. Nat Rev Cancer 11(12): 865-878.

Chacon M, Reichardt P, Gu J, Nishida T, Barrios CH, Blay J, Reichardt A, Blackstein ME, Casali PG, Kang Y (2010) The GOLD reGISTry: A global observational registry collecting longitudinal data on patients with advanced GIST-Second annual summary. J Clin Oncol 28(15s): abstr no 10092

DeMatteo RP, Lewis JJ, Leung D, Mudan SS (2000) Two hundred gastrointestinal stromal tumors: Recurrence patterns and prognostic factors for survival. Ann Surg 231(1): 51-58.

Demetri GD, van Oosterom AT, Garrett CR, Blackstein ME, Shah MH, Verweij J, McArthur G, Judson IR, Heinrich MC, Morgan JA, Desai J, Fletcher CD, George S, Bello CL, Huang X, Baum CM, Casali PG (2006) Efficacy and safety of sunitinib in patients with advanced gastrointestinal stromal tumour after failure of imatinib: a randomised controlled trial. Lancet 368(9544): 1329-1338.

Fletcher CD, Berman JJ, Corless C, Gorstein F, Lasota J, Longley BJ, Miettinen M, O'Leary TJ, Remotti H, Rubin BP, Shmookler B, Sobin LH, Weiss SW (2002) Diagnosis of gastrointestinal stromal tumors: A consensus approach. Hum Pathol 33(5): 459-465.

Gliklich RE, Dreyer NA (2010) Registries for Evaluating Patient Outcomes: A User's Guide. 2nd edn (Prepared by Outcome DEcIDE Center [Outcome Sciences, Inc. d/b/a Outcome] under Contract No. HHSA29020050035I TO3.) AHRQ Publication No.10-EHC049) Agency for Healthcare Research and Quality: Rockville, MD, USA.

Heinrich MC (2010) Imatinib treatment of metastatic GIST; don't stop (believing). Lancet Oncol 11(10): 910-911.

Hirota S, Isozaki K, Moriyama Y, Hashimoto K, Nishida T, Ishiguro S, Kawano K, Hanada M, Kurata A, Takeda M, Muhammad Tunio G, Matsuzawa Y, Kanakura Y, Shinomura Y, Kitamura Y (1998) Gain of function mutations of c-kit in human gastrointestinal stromal tumours. Science 279(5350): 577-580.

Joensuu H, Roberts PJ, Sarlomo-Rikala M, Andersson LC, Tervahartiala P, Tuveson D, Silberman S, Capdeville R, Dimitrijevic S, Druker B, Demetri GD
(2001) Effect of tyrosine kinase inhibitor STI571 in a patient with a metastatic gastrointestinal stromal tumor. $N$ Engl J Med 344(14): 1052-1056.

Kim KM, Kang DW, Moon WS, Park JB, Park CK, Sohn JH, Jeong JS, Cho MY, Jin SY, Choi JS, Kang DY. Gastrointestinal Stromal Tumor Committee; Korean Gastrointestinal Pathology Study Group (2005) Gastrointestinal stromal tumors in Koreans: its incidence and the clinical, pathologic and immunohistochemical findings. J Korean Med Sci 20(6): 977-984.

Kim SY, Janeway K, Pappo A (2010) Pediatric and wildtype gastrointestinal stromal tumour (GIST): new therapeutic approaches. Curr Opin Oncol 22(4): 347-350.

Miettinen M, Lasota J (2001) Gastrointestinal stromal tumors - definition, clinical, histological, immunohistochemical, and molecular genetic features and differential diagnosis. Virchows Arch 438(1): 1-12.

Miettinen M, Lasota J (2006a) Gastrointestinal stromal tumors: review on morphology, molecular pathology, prognosis, and differential diagnosis. Arch Pathol Lab Med 130(10): 1466-1478.

Miettinen M, Lasota J (2006b) Gastrointestinal stromal tumors: Pathology and prognosis at different sites. Semin Diagn Pathol 23(2): 70-83.

National Institute for Clinical Excellence (2004) Imatinib for the treatment of unresectable and/or metastatic gastrointestinal stromal tumours. Available at www.nice.org.uk/nicemedia/live/11548/32969/32969.pdf (Accessed February 2013).

National Institute for Health and Clinical Excellence (2009) Sunitinib for the treatment of gastrointestinal stromal tumours. Available at www.nice. org.uk/nicemedia/pdf/TA179Guidance.pdf (Accessed February 2013).

Nilsson B, Bümming P, Meis-Kindblom JM, Odén A, Dortok A, Gustavsson B, Sablinska K, Kindblom LG (2005) Gastrointestinal stromal tumors: the incidence, prevalence, clinical course, and prognostication in the preimatinib mesylate era-a population-based study in western Sweden. Cancer 103(4): 821-829.

Pisters PWT, Blanke CD, von Mehren M, Picus J, Sirulnik A, Stealey E, Trent JC. reGISTry Steering Committee (2011) A USA registry of gastrointestinal stromal tumor patients: changes in practice over time and differences between community and academic practices. Ann Oncol 22(11): 2523-2529.

Tryggvason G, Gíslason HG, Magnússon MK, Jónasson JG (2005) Gastrointestinal stromal tumors in Iceland, 1990-2003: The Icelandic GIST study, a population-based incidence and pathologic risk stratification study. Int J Cancer 117(2): 289-293.

West R, Cohen C, Xin C, Rubin BP, Subramanian S, Montgomery K, Zhu S, Ball CA, Nielsen TO, Patel R, Goldblum JR, Brown PO, Heinrich MC, van de Rijn M (2004) The novel marker, DOG1, is expressed ubiquitously in gastrointestinal stromal tumors irrespective of KIT or PDGFRA mutation status. Am J Surg Path 165(1): 107-113.

This work is published under the standard license to publish agreement. After 12 months the work will become freely available and the license terms will switch to a Creative Commons AttributionNonCommercial-Share Alike 3.0 Unported License. 